\title{
Poda da parte aérea, visando melhor qualidade de mudas de Pinus taeda, em Caçador, SC
}

\author{
Saulo Jorge Téo ${ }^{1}$, Alan Marcon ${ }^{1}$, Reinaldo Hoinacki da Costa² \\ ${ }^{1}$ Universidade do Oeste de Santa Catarina, Rua Dirceu Giordani, 696, CEP 89820-000, Xanxerê, SC, Brasil \\ 2Juliana Florestal Ltda., Rua Padre Feijó, 241, CEP 89500-000, Caçador, SC, Brasil
}

"Autor correspondente:
sauloteo@yahoo.com.br

Termos para indexação:

Intensidade de poda

Relação h/d

Mortalidade

\section{Index terms:}

Pruning intensity

$\mathrm{h} / \mathrm{d}_{\mathrm{n}}$ relation

Mortality

Histórico do artigo:

Recebido em 17/12/2012

Aprovado em 10/03/2014

Publicado em 04/04/2014
Resumo - O objetivo desta pesquisa foi avaliar os efeitos da poda da parte aérea sobre a sobrevivência, altura (h), diâmetro de colo $\left(\mathrm{d}_{\mathrm{c}}\right)$ e relação $\mathrm{h} / \mathrm{d}_{\mathrm{c}}$, de mudas de Pinus taeda L., no Município de Caçador, SC, visando obter mudas de melhor qualidade. Foram testados seis diferentes tipos e intensidades de podas. Os dados foram submetidos ao teste de homogeneidade das variâncias (Teste de Bartlett), à análise de variâncias (ANOVA) e ao teste de comparação de médias de Tukey $(\alpha=5 \%)$. Aos 60 dias após a instalação do experimento, não houve mortalidade de mudas. A retirada da porção apical das mudas resultou em formação de brotações laterais, diminuição significativa das alturas e da relação $\mathrm{h} / \mathrm{d}$ das mudas, melhorando o padrão de qualidade das mudas. Por outro lado, a retirada das acículas na porção inferior das mudas causou aumento significativo das alturas, mas não afetou a relação $h / \mathrm{d}_{\mathrm{c}}$. Nenhum dos tratamentos resultou em aumento significativo do diâmetro de colo. Entretanto, a poda mais severa da parte aérea (corte da porção apical + remoção de $2 / 3$ das acículas) causou redução significativa do diâmetro de colo e, consequentemente, da qualidade das mudas.

\section{Above ground pruning, aiming better quality of seedlings of Pinus taeda, in Caçador, SC}

\begin{abstract}
The objective of this paper was to evaluate the effects caused by the above ground pruning over the survival, height $(\mathrm{h})$, neck diameter $\left(\mathrm{d}_{\mathrm{n}}\right)$ and $\mathrm{h} / \mathrm{d}_{\mathrm{n}}$ relation, of Pinus taeda L. seedlings, in the municipal district of Caçador, Santa Catarina State, Brazil, aiming to obtain seedlings of better quality. Six different types and intensities of pruning were tested. The data were analyzed by the variances homogeneity test (Bartlett's test), variance analysis (ANOVA) and, by the Tukey's test of means comparison ( $\alpha=$ $5 \%$ ). Sixty days after the experiment installation, there was no seedlings mortality; the removal of the upper portion of the seedlings showed abundant formation of side shoots, significant decrease of seedling heights and $\mathrm{h} / \mathrm{d}_{\mathrm{n}}$ relation, improving the quality pattern of the seedlings. On the other hand, the removal of the lower portion of seedlings leaves caused significant increase of heights, but did not affect the $h / d_{n}$ relation. None of the treatments resulted in significant increase in neck diameter. However, the most intensive above ground pruning (upper portion removal + removal of the $2 / 3$ of the lower portion of leaves) caused significant reduction of the neck diameter and, consequently, of the seedlings quality.
\end{abstract}




\section{Introdução}

Sabe-se que para se concretizar um empreendimento florestal de qualidade são necessários grandes investimentos, pois a formação de novos povoamentos ou a reforma de áreas anteriormente plantadas são tarefas difíceis e onerosas. Como o reflorestamento é um investimento de longo prazo, a escolha das mudas deve ser cuidadosa para possibilitar que o povoamento se desenvolva bem, de forma a não acarretar perdas no futuro. Segundo Gomes et al. (1978), a qualidade das mudas irá definir o sucesso ou não de um reflorestamento. Uma muda de qualidade garantirá o êxito, assim como um menor índice de mortalidade e necessidade de posterior replantio.

Dentre os parâmetros morfológicos de classificação da qualidade de mudas, os mais estudados e discutidos são a altura da parte aérea $(\mathrm{h})$, o diâmetro do colo $\left(\mathrm{d}_{\mathrm{c}}\right)$, a relação altura da parte aérea e diâmetro do colo (h/ $\mathrm{d}_{\mathrm{c}}$ ) e a massa, tanto da parte aérea, como das raízes das mudas (Carneiro, 1995). De acordo com Binotto (2007), a fitomassa seca de raiz foi a variável mais relacionada com a qualidade das mudas, porém sua obtenção necessita da destruição da muda, sendo o diâmetro do colo a variável mais propícia para indicar a qualidade de mudas. Além dessas, segundo Gomes et al. (2002), a altura apresentou boa contribuição relativa à qualidade das mudas, sendo um parâmetro de fácil obtenção e não destrutivo.

Os parâmetros em que os pesquisadores fundamentam-se para conceituar a qualidade de mudas, sejam eles morfológicos ou fisiológicos, são fortemente influenciados pelas técnicas de produção, notadamente pela densidade, podas, espécie de fungos e grau de colonização de micorrizas, fertilidade do substrato e volume disponível para cada planta. Em se tratando de mudas em recipientes, há que se considerar, além dos aspectos citados, a influência da forma, dimensões e do material que compõe a sua parede (Carneiro, 1995).

De acordo com Carneiro (1995), a poda é a remoção de uma parte da planta, com a finalidade de melhorá-la em algum aspecto que seja de interesse do cultivador. A poda, tanto da parte aérea, como da radicular, pode apresentar resultados favoráveis ao desenvolvimento das mudas, sendo que as principais finalidades deste tipo de procedimento são: promover o balanço da parte aérea em comparação com o sistema radicular; aumentar a percentagem de sobrevivência das mudas no campo ou no viveiro; propiciar a produção de mudas mais robustas; estimular a produção de raízes novas com maior capacidade de absorção de nutrientes; retardar o crescimento em altura, o qual será posteriormente recuperado no campo, e aumentar a vida útil da muda.

A poda do sistema radicular de mudas é a mais frequentemente utilizada no Brasil, para diferentes essências florestais, a exemplo dos trabalhos de Malinovski (1977), Souza et al. (2006), Castro Faria et al. (2007) e Freitas et al. (2009). Já os estudos sobre poda da parte aérea de mudas são incomuns, sobretudo no Brasil. Este tipo de poda vem sendo aplicado, tanto para aumentar a vida útil de mudas no viveiro, como para melhorar a sobrevivência e desempenho das mudas após o plantio no campo (Duryea, 1990; McCreary \& Tecklin, 1993; Gilman et al., 2006).

De acordo com McCreary \& Tecklin (1993), quando o crescimento das raízes da muda é restrito, estas se tornam muito pequenas em relação à parte aérea, para fornecer água e nutrientes. No momento do plantio definitivo no campo, tais mudas podem ter crescimento muito lento ou, até mesmo, morrer. Para muitas espécies florestais, a poda da parte aérea no viveiro tem sido utilizada para reduzir e restaurar o equilíbrio entre a parte aérea e o sistema radicular.

Duryea (1990) testou os efeitos da poda da parte aérea em mudas de Pinus elliottii Engelm. e constatou que, após três anos de plantio, as mudas podadas não apresentaram diferenças de crescimento e sobrevivência, com relação às mudas não podadas. South (1998), estudando a poda da parte aérea de mudas de $P$. taeda, reportou que não houve diferenças quanto ao crescimento das mudas, entretanto, a sobrevivência das mudas submetidas à poda aumentou em $6 \%$ no viveiro.

McCreary \& Tecklin (1993) e Gilman et al. (2006) avaliaram a poda da parte aérea, combinada com diferentes localidades e épocas de plantio, para mudas de Quercus douglasii Hook et Arn. e Quercus virginiana Mill., nos Estados Unidos. Após um período de avaliação, os autores recomendaram a poda da parte aérea das mudas para melhorar seu desempenho no campo.

Existe uma demanda crescente de mudas com boa qualidade para formação de povoamentos florestais comerciais. Para isto, a busca pela melhoria das técnicas silviculturais no desenvolvimento das mudas deve ser contínua. 
Portanto, o objetivo desta pesquisa foi avaliar os efeitos advindos da poda da parte aérea sobre a sobrevivência, altura $(\mathrm{h})$, diâmetro de colo $\left(\mathrm{d}_{\mathrm{c}}\right)$ e relação $\mathrm{h} / \mathrm{d}_{\mathrm{c}}$, de mudas de Pinus taeda L., no Município de Caçador, SC, visando obter mudas de melhor qualidade.

\section{Material e métodos}

A área de estudo foi o viveiro da empresa Juliana Florestal Ltda., localizado no Município de Caçador, Estado de Santa Catarina. Embora Caçador apresente grande parte de seu território com altitudes superiores a $1.000 \mathrm{~m}$ acima do nível do mar, chegando a $1.390 \mathrm{~m}$ na Serra da Taquara Verde, a área de estudo localiza-se em altitude inferior a $1.000 \mathrm{~m}$, na bacia do Rio do Peixe, ao sul da área urbana de Caçador (IBGE, 1973; 2009; 2011).

O clima mesotérmico é subtropical úmido sem estação seca, com verões frescos, segundo a classificação de Köppen. É uma região de ocorrência de clima tipo $\mathrm{Cfb}$, isto é, temperado úmido, com geadas severas, temperatura média do mês mais quente inferior a $22^{\circ} \mathrm{C}$, sendo que nos meses de inverno, as médias variam entre $6{ }^{\circ} \mathrm{C}$ e $8{ }^{\circ} \mathrm{C}$ (Caldato et al., 1999). Conforme informações meteorológicas da Estação Experimental de Caçador, a temperatura média anual é de $16,5^{\circ} \mathrm{C}$, a precipitação média anual é de $1.608 \mathrm{~mm}$ e a umidade relativa do ar média anual é de $77 \%$, com ocorrência de 26 geadas por ano, em média (Kurasz, 2005).

A semeadura das mudas foi realizada em tubetes de polipropileno com $50 \mathrm{~cm}^{3}$ de volume e seis estrias, que foram acondicionados em bandejas de plástico com capacidade para 216 tubetes. Foi utilizado substrato composto de casca de pinus, vermiculita, carvão e calcário.

No viveiro, foram realizados seis diferentes tratamentos: 1 - controle, sem a realização de poda; 2 corte de $5 \mathrm{~cm}$ da porção apical da muda; 3 - remoção de $2 / 3$ das acículas na porção inferior da muda; 4 - remoção de $1 / 3$ das acículas na porção inferior da muda; 5 - corte de $5 \mathrm{~cm}$ da porção apical + remoção de $1 / 3$ das acículas na porção inferior da muda; 6 - corte de $5 \mathrm{~cm}$ da porção apical + remoção de $2 / 3$ das acículas na porção inferior da muda.

Após 90 dias da semeadura, foram realizadas as podas e uma medição da altura e diâmetro de colo das mudas. A partir de então, a cada 15 dias uma nova medição da altura da muda, diâmetro de colo e porcentagem de sobrevivência foi realizada, até 150 dias de vida. A altura da muda (h), desde o colo até o ápice, foi mensurada com uma régua graduada. $\mathrm{O}$ diâmetro de colo da muda $\left(\mathrm{d}_{\mathrm{c}}\right)$ foi obtido com o auxílio de um paquímetro, graduado em mm. A partir das medições da altura e do diâmetro de colo, foi calculada a relação $\mathrm{h} / \mathrm{d}_{\mathrm{c}}$. A porcentagem de sobrevivência foi quantificada pela diferença entre o número de mudas no início da instalação do experimento e em cada período de avaliação.

O delineamento experimental utilizado foi inteiramente casualizado, composto por seis tratamentos (tipos de poda da parte aérea das mudas), quatro repetições e 14 mudas por unidade experimental.

Para a análise de variância dos dados de altura da parte aérea, diâmetro do colo e relação $\mathrm{h} / \mathrm{d}_{\mathrm{c}}$, considerouse o delineamento estatístico inteiramente casualizado. Antes da análise de variância, aplicou-se o teste de homogeneidade das variâncias dos tratamentos (teste de Bartlett), para cada variável analisada. Para verificação das diferenças entre as médias de cada tratamento, aplicou-se o teste de comparação de médias de Tukey $(\alpha=5 \%)$.

\section{Resultados e discussão}

Com base no desenvolvimento das mudas após a poda da parte aérea, verificou-se um crescimento gradual e contínuo, tanto em diâmetro como em altura. Os valores médios de altura e diâmetro do colo aos 150 dias, considerando-se todos os tratamentos do experimento, foram de $21,35 \mathrm{~cm}$ e $2,4 \mathrm{~mm}$, respectivamente. Já a relação $\mathrm{h} / \mathrm{d}_{\mathrm{c}}$ variou pouco durante o período, permanecendo entre 8,54 e 9,19 (Figura 1). Geralmente, no viveiro deste estudo, as mudas são consideradas aptas para serem levadas para o campo quando atingem um diâmetro acima de $2 \mathrm{~mm}$ e altura de $15 \mathrm{~cm}$ a $25 \mathrm{~cm}$. Portanto, ao final do experimento, as mudas estavam dentro dos padrões estabelecidos.

Carneiro (1995) recomendou parâmetros que definem qualidade de mudas do gênero Pinus produzidas em recipientes com altura de $15 \mathrm{~cm}$ a $25 \mathrm{~cm}$, porém diâmetro de colo mínimo de 3,7 mm. Já Pezzutti \& Caldato (2011), considerando diversos autores, definiram o diâmetro do colo entre $2,5 \mathrm{~cm}$ e $3 \mathrm{~mm}$ como parâmetro mínimo de qualidade de mudas de Pinus sp. produzidas em recipientes. 


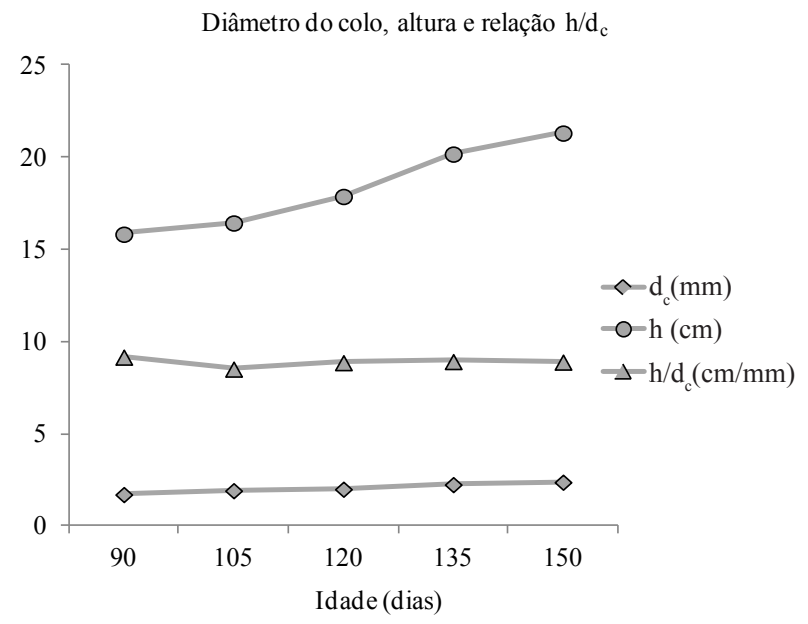

Figura 1. Diâmetro do colo, altura e relação $h / d_{c}$ médios para todos os indivíduos de um experimento de poda da parte aérea de mudas de Pinus taeda, em Caçador, SC.

Gomes et al. (2003) consideraram que quanto menor o valor da relação $h / d_{c}$, maior será a capacidade das mudas sobreviverem e se estabelecerem no campo. Rossi (2005), Carneiro \& Brito (1992) e Novaes et al. (2001) encontraram valores de relação $\mathrm{h} / \mathrm{d}_{\mathrm{c}}$ para mudas de Pinus taeda menores do que o valores encontrados neste estudo.

Por outro lado, em trabalho sobre crescimento e nutrição de mudas de Pinus taeda no Estado do Rio Grande do Sul, Thomas (2007) observou, aos 180 dias de idade, valores médios para altura, diâmetro de colo e relação $\mathrm{h} / \mathrm{d}_{\mathrm{c}}$, de $29,0 \mathrm{~cm}, 2,76 \mathrm{~mm}$ e 10,7 , respectivamente.

Até os 150 dias após a semeadura, não houve taxa de mortalidade para as mudas de $P$. taeda, que sofreram diferentes tipos de poda da parte aérea. Além disso, as mudas submetidas aos tratamentos com poda da porção apical apresentaram grande formação de brotações laterais.

As variâncias dos tratamentos foram consideradas homogêneas pelo teste de Bartlett, atendendo às condicionantes para que se possa realizar a análise de variância. Os resultados de $X_{\text {corr }}^{2}$ do teste de Bartlett para as variáveis diâmetro do colo, altura e relação $h / d_{c}$ foram de $5,22^{\text {ns }}, 6,29^{\text {ns }}$ e $3,32^{\text {ns }}$, respectivamente.

De acordo com os valores de $F_{\text {calc }}$ e níveis de significância ( $p$-level), apresentados na Tabela 1, observa-se que os diferentes tipos de poda da parte aérea das mudas de Pinus taeda exerceram efeito significativo no desenvolvimento das mudas em diâmetro do colo, altura e relação $\mathrm{h} / \mathrm{d}_{\mathrm{c}}$.
Tabela 1. Resultados de $F_{\text {calc }}$ e níveis de significância ( $p$-level) para diâmetro do colo, altura e relação $\mathrm{h} / \mathrm{d}_{\mathrm{c}}$ aos 150 dias, do experimento de poda da parte aérea das mudas de Pinus taeda, em Caçador, SC.

\begin{tabular}{cccc}
\hline \multicolumn{3}{c}{ Variáveis } \\
\hline$F_{\text {calc }}$ & $\mathrm{d}_{\mathrm{c}}(\mathrm{mm})$ & $\mathrm{h}(\mathrm{cm})$ & $\mathrm{h} / \mathrm{d}_{\mathrm{c}}$ \\
$p$-level & $5,79 \times 17^{*}$ & $277,57^{*}$ & $131,67^{*}$ \\
\hline ns = não significativo $*$ * significativo a $1 \%$.
\end{tabular}

ns = não significativo; $*$ = significativo a $1 \%$.

Aos 150 dias de idade, os tratamentos 1, 2, 3 e 5 apresentaram mudas com os maiores valores de diâmetro de colo (Tabela 2). É possível verificar ainda que o diâmetro de colo das mudas submetidas ao tratamento 4 não foi diferente do diâmetro de colo das mudas submetidas aos tratamentos $1,3,5$ e 6 .

Tabela 2. Resultados do teste de Tukey para o diâmetro de colo (mm), altura $(\mathrm{cm})$ e relação $\mathrm{h} / \mathrm{dc}$, aos 150 dias, considerando os diferentes tratamentos de poda da parte aérea de mudas de Pinus taeda, na região de Caçador, SC.

\begin{tabular}{cccccc}
\hline \multicolumn{2}{c}{$\begin{array}{c}\text { Diâmetro de colo } \\
(\mathbf{m m})\end{array}$} & \multicolumn{2}{c}{ Altura (cm) } & \multicolumn{2}{c}{ Relação h/d } \\
\hline Tratamentos & Média & Tratamentos & Média & Tratamentos & Média \\
\hline 2 & $2,50 \mathrm{a}$ & 4 & $24,98 \mathrm{a}$ & 3 & $10,13 \mathrm{a}$ \\
5 & $2,47 \mathrm{ab}$ & 3 & $23,83 \mathrm{~b}$ & 4 & $10,13 \mathrm{a}$ \\
1 & $2,42 \mathrm{ab}$ & 1 & $23,72 \mathrm{~b}$ & 1 & $9,82 \mathrm{a}$ \\
3 & $2,39 \mathrm{ab}$ & 6 & $20,16 \mathrm{c}$ & 6 & $8,91 \mathrm{~b}$ \\
4 & $2,35 \mathrm{bc}$ & 5 & $18,46 \mathrm{~d}$ & 5 & $7,74 \mathrm{c}$ \\
6 & $2,26 \mathrm{c}$ & 2 & $16,97 \mathrm{e}$ & 2 & $6,79 \mathrm{~d}$ \\
\hline
\end{tabular}

Tratamentos: $1=$ controle, sem a realização de poda; $2=$ corte de $5 \mathrm{~cm}$ da porção apical da muda; 3 = remoção de $2 / 3$ das acículas na porção inferior da muda; 4 = remoção de $1 / 3$ das acículas na porção inferior da muda; $5=$ corte de $5 \mathrm{~cm}$ da porção apical + remoção de $1 / 3$ das acículas na porção inferior da muda; 6 = corte de $5 \mathrm{~cm}$ da porção apical + remoção de $2 / 3$ das acículas na porção inferior da muda. Médias seguidas de letras iguais não diferem estatisticamente ao nível de significância de $5 \%(\alpha=0,05)$, pelo teste de Tukey.

Nenhum dos tratamentos que compreendem aplicação de poda às mudas de Pinus taeda resultaram em aumento estatisticamente significativo do diâmetro de colo, quando comparados ao tratamento controle (mudas sem aplicação de poda da parte aérea). No entanto, o tratamento 6 apresentou diâmetro de colo significativamente menor quando comparado aos tratamentos 1, 2, 3 e 5. Dessa forma, a técnica de poda mais severa (tratamento 6) aplicada neste estudo causou redução significativa do diâmetro de colo das mudas. 
A classificação morfológica de mudas florestais pode ser feita considerando-se a altura da parte aérea, o diâmetro do colo, a relação $\mathrm{h} / \mathrm{d}_{\mathrm{c}}$, o peso de matéria seca e verde e rigidez da haste. Entretanto, o diâmetro do colo tem sido reconhecido como um dos melhores, senão o melhor dos indicadores de padrão de qualidade, pois está associado ao desenvolvimento mais acentuado da muda, principalmente, do sistema radicular (Sturion \& Antunes, 2000). Por sua vez, o desenvolvimento radicular constitui o fator mais importante na sobrevivência e crescimento de pinus no primeiro ano após o plantio (Dougherty \& Gresham, 1988).

Diferente dos resultados encontrados neste estudo, Duryea \& Omi (1987) e Duryea (1990) estudando mudas de Pseudotsuga menziesii Franco e Pinus elliottii, respectivamente, observaram que as mudas submetidas à poda da parte aérea apresentaram diâmetros menores, quando comparadas às mudas não podadas.

A Tabela 2 demonstra que, após 150 dias da semeadura, o tratamento 4 resultou em mudas com os maiores valores de altura. Foi observada diferença significativa entre os valores de altura do tratamento 4 e todos os demais tratamentos pelo teste de Tuckey (5\%). O tratamento 3 e o tratamento 1 (controle), são os únicos tratamentos estatisticamente iguais, representando o grupo com alturas imediatamente inferiores ao tratamento $4 \mathrm{e}$ superiores aos demais. Portanto, pode-se afirmar que as podas que consistiam em remoção das acículas da porção inferior da muda exerceram efeito positivo no crescimento em altura das mudas de $P$. taeda. Por outro lado, as mudas que sofreram corte da sua porção apical apresentaram valores de altura significativamente menores, concordando com os resultados observados por Duryea (1990) e South (1998), para Pinus elliottii e Pinus taeda, respectivamente.

Ao se avaliar a altura das mudas submetidas à poda da parte aérea, foi possível notar a formação de intensa brotação lateral, principalmente para os tratamentos 2 , 5 e 6 . O menor crescimento em altura, em resposta à poda das mudas, pode ser atribuído ao aumento de brotações laterais, comportamento também observado por Duryea \& Omi (1987) e Duryea (1990), para Pseudotsuga menziesii e Pinus elliottii, respectivamente. De acordo com Carneiro (1995), em mudas de Pinus spp., a poda aérea provoca o aparecimento de alguns brotos apicais, um dos quais, com o passar do tempo, assume a predominância com relação aos demais.
$\mathrm{Na}$ Tabela 2, verifica-se que os tratamentos 3, 4 e o tratamento controle apresentaram os maiores valores de relação $h / d_{c}$ e não diferiram entre si. Os tratamentos 2,5 e 6 apresentam diferença estatística significativa entre os valores de relação $\mathrm{h} / \mathrm{d}_{\mathrm{c}}$, teste de Tukey (5\%). Observase que o tratamento 2 , além de ser estatisticamente diferente, apresenta uma média de relação $\mathrm{h} / \mathrm{d}_{\mathrm{c}}$ inferior aos demais.

Rossi (2005) estudou o crescimento e qualidade de mudas de Pinus taeda, submetidas à poda química de raízes e encontrou médias de relação $\mathrm{h} / \mathrm{d}_{\mathrm{c}}$ que variaram de 7,9 a 8,6, aos 8 meses de idade. Carneiro \& Brito (1992), estudando uma nova metodologia para produção mecanizada de mudas de Pinus taeda em recipientes com raízes laterais podadas, realizaram avaliações aos 11 meses após a semeadura e encontraram uma média de relação $\mathrm{h} / \mathrm{d}_{\mathrm{c}}$, que variou de 5,9 a 8,2. Os valores da relação $\mathrm{h} / \mathrm{d}_{\mathrm{c}}$ encontrados por esses autores são menores do que os valores encontrados para a maioria dos tratamentos deste estudo. Porém, deve-se considerar que as mudas deste estudo foram avaliadas aos cinco meses de idade.

No entanto, Novaes et al. (2001) e Reis (2006) encontraram valores de relação $\mathrm{h} / \mathrm{d}_{\mathrm{c}}$ variando entre 6,11 e 7,25 e entre 5,98 e 6,51, respectivamente, para mudas de Pinus taeda aos 180 dias após a semeadura e de Pinus elliottii aos 150 dias após a semeadura. Esses valores de relação $h / d_{c}$ são semelhantes aos valores encontrados para os tratamentos 2 e 5 (Tabela 2).

\section{Conclusões}

Não houve taxa de mortalidade para as mudas de Pinus taeda, que sofreram diferentes tipos de poda da parte aérea, na região de Caçador, SC.

A técnica de poda da parte aérea mais severa aplicada às mudas de Pinus taeda causou redução significativa do diâmetro de colo e, consequentemente, da qualidade das mudas. Por outro lado, nenhuma das técnicas de poda aplicada resultou em aumento significativo do diâmetro de colo das mudas de Pinus taeda.

Embora as alturas das mudas não tenham excedido os limites definidos pelos padrões de qualidade, a retirada da porção inferior das acículas das mudas de Pinus taeda resultou em aumento significativo das alturas, enquanto que a remoção da porção apical das mudas de Pinus taeda resultou em diminuição significativa das alturas.

Pesq. flor. bras., Colombo, v. 34, n. 77, p. 57-62, jan./mar. 2014 
A retirada da porção inferior das acículas de Pinus taeda não resultou em diferença significativa na relação $\mathrm{h} / \mathrm{d}_{\mathrm{c}}$ das mesmas. Por outro lado, a remoção da porção apical das mudas de Pinus taeda resultou em diminuição significativa dos valores da relação $\mathrm{h} / \mathrm{d}_{\mathrm{c}}$, melhorando o padrão de qualidade das mudas.

\section{Referências}

BINOTTO, A. F. Relação entre variáveis de crescimento e o índice de qualidade de Dickson em mudas de Eucalyptus grandis W. Hill ex Maid e Pinus elliottii var. elliottii - Engelm. 2007. 54 f. Dissertação (Mestrado em Engenharia Florestal) - Universidade Federal de Santa Maria, Santa Maria, RS.

CALDATO, S. L.; LONGHI, S. J.; FLOSS, P. A. Estrutura populacional de Ocotea porosa (Lauraceae) em uma Floresta Ombrófila Mista, em Caçador (SC). Ciência Florestal, Santa Maria, RS, v. 9, n. 1, p. 89-101, 1999.

CARNEIRO, J. G. A.; BRITO, M. A. R. Nova metodologia para produção mecanizada de mudas de Pinus taeda L. em recipientes com raízes laterais podadas. Floresta, Curitiba, v. 22, n. 1/2, p. 63-77, 1992.

CARNEIRO, J. G. A. Produção e controle de qualidade de mudas florestais. Curitiba: UFPR-FUPEF; Campos: UNEF, 1995. 451 p.

CASTRO FARIA, A. B.; HIRANO, E.; PORTELA, O.; VOSGERAU, J. L.; NOGUEIRA, A. C. Poda radicial em mudas de Rheedia gardneriana. Scientia Agraria, Curitiba, v. 8, n. 1, p. 99-101, 2007.

DOUGHERTY, P. M.; GRESHAM, C. A. Conceptual analysis of southern pine plantation establishment an early growth. Southern Journal of Applied Forestry, Bethesda, v. 12, n. 3, p. 160-166, 1988.

DURYEA, M. L. Nursery fertilization and top pruning of slash pine seedlings. Southern Journal of Applied Forestry, Bethesda, v. 14, n. 2, p. 73-76, 1990.

DURYEA, M. L.; OMI, S. K. Top pruning Douglas-fir seedlings: morphology, physiology, and field performance. Canadian Journal of Forest Research, Toronto, v. 17, n. 11, p. 1371-1378, 1987.

FREITAS, T. A. S. de; BARRoso, D. G.; SOUZA, L. S. CARNEIRO, J. G. A. Efeito da poda de raízes sobre o crescimento das mudas de eucalipto. Ciência Florestal, Santa Maria, RS, v. 19, n. 1, p. 1-6. 2009.

GILMAN, E. F.; ANDERSON, P. J.; HARCHICK, C. Pruning lower branches of live oak (Quercus virginiana Mill.) cultivars and seedlings during nursery production: balancing growth and efficiency. Journal of Environmental Horticulture, Washington, DC, v. 24, n. 4, p. 201-206. 2006.

GOMES, J. M.; BRANDI, R. M.; CANO, M. A. O.; SOUZA, A. P. de. Efeitos do sal, antitranspirante e poda no endurecimento a seca de mudas de Eucalyptus grandis W. Hill ex Maiden. Floresta, Curitiba, v. 9 , n. 2, p. 18-24, 1978.

GOMES, J. M.; COUTO, L.; LEITE, H. G.; XAVIER, A.; GARCIA, S. L. R. Crescimento de mudas de Eucalyptus grandis em diferentes tamanhos de tubetes e fertilização N-P-K. Revista Árvore, Viçosa, MG, v. 27, n. 2, p. 113-127, 2003.
GOMES, J. M.; COUTO, L.; LEITE, H. G.; XAVIER, A.; GARCIA, S. L. R. Parâmetros morfológicos na avaliação da qualidade de mudas de Eucalyptus grandis. Revista Árvore, Viçosa, MG, v. 26, n. 6, p. 655-664, 2002.

IBGE. Caçador. Rio de Janeiro, 1973. Carta topográfica. Escala: $1: 100.000$.

IBGE. Mapa físico do Estado de Santa Catarina. Rio de Janeiro, 2011. Mapa físico. Escala: 1:800.000.

IBGE. Mapa político do Estado de Santa Catarina. Rio de Janeiro, 2009. Mapa político. Escala: 1:800.000.

KURASZ, G. Sistema de informações geográficas aplicado ao zoneamento ambiental da Reserva Florestal Embrapa/Epagri, Caçador/SC. 2005. 146 f. Dissertação (Mestrado em Ciências Florestais) - Universidade Federal do Paraná, Curitiba.

MALINOVSKI, J. R. Métodos de poda radicular em Araucaria angustifolia (Bert.) O. Ktze. e seus efeitos sobre a qualidade de mudas de raiz nua. Floresta, Curitiba, v. 8, n. 1, p. 85-88, 1977.

McCREARY, D.; TECKLIN, J. Top pruning improves field performance of blue oak seedlings. Tree Planters' Notes, Washington, DC, v. 44, n. 2, p. 73-77, 1993.

NOVAES, A. B. de; CARNEIRO, J. G. A.; BARRoso, D. G.; LELES, P. S. S. Desempenho de mudas de Pinus taeda produzidas em raiz nua e em dois tipos de recipientes, 24 meses após o plantio. Floresta, Curitiba, v. 31, n. 1/2, p. 62-71, 2001.

PEZZUTTI, R. V.; CALDATO, S. L. Sobrevivência e crescimento inicial de mudas de Pinus taeda L. com diferentes diâmetros do colo. Ciência Florestal, Santa Maria, RS, v. 21, n. 2, p. 355-362, 2011.

REIS, E. R. dos. Variação espacial e temporal dos parâmetros morfológicos em mudas de pinus e eucalipto. 2006. $49 \mathrm{f}$. Dissertação (Mestrado em Engenharia Florestal) - Universidade Federal de Santa Maria, Santa Maria, RS.

ROSSI, V. L. Crescimento e qualidade de mudas de Pinus taeda L. submetidas à poda química de raízes com cobre e ethefon. 2005. 40 f. Dissertação (Mestrado em Produção Vegetal) - Universidade do Estado de Santa Catarina, Lages.

SOUTH, D. B. Needle-clipping longleaf pine and top-pruning loblolly pine in bareroot nurseries. Southern Journal of Applied Forestry, Bethesda, v. 22, n. 4, p. 235-240, 1998.

SOUZA, G. L. F. M.; RIBEIRO, M. C. C.; CARDOSO, E. A.; PIRES, G. S.; SOUZA, P. A. de. Efeito da época de poda do sistema radicular no crescimento de mudas de Algarobeira. Revista Caatinga, Mossoró, v. 19, n. 3, p. 250-254, 2006.

STURION, J. A.; ANTUNES, J. B. M. Produção de mudas de espécies florestais. In: GALVÃO, A. P. M. Reflorestamento de propriedades rurais para fins produtivos e ambientais: um guia para ações municipais e regionais. Colombo: Embrapa Florestas, 2000. p. $125-150$.

THOMAS, R. Crescimento e nutrição de mudas de Pinus taeda $\mathbf{L}$. no Estado do Rio Grande do Sul. 2007. 64 f. Dissertação (Mestrado em Engenharia Florestal) - Universidade Federal de Santa Maria, Santa Maria. 